\title{
Untersuchung des Absorptionsspectrums von Indigotin, Diamidoindigo und Tetrazoindigo \\ von
}

Josef Maria Eder in Wien.

(Mit 1 Textfigur.)

(Vorgelegt in der Sitzung am 23. October 1902.)

Herr Prof. Dr. Paul Friedländer in Wien übermittelte mir zum Zwecke spectralanalytischer Untersuchung von ihm selbst hergestellte Proben von Indigotin, respective indigodisulfosaurem Natrium, von Diamidoindigo in Form von gelöstem salzsaurem Diamidoindigo, aus welchem letzteren nach Prof. Friedländer's Angaben durch Zusatz von Kaliumnitrit und Spuren von Salzsäure Tetrazoindigo hergestellt wurde.

Die spectroskopische Untersuchung dieser Lösungen nahm ich mittels eines Krüss'schen Universalspectralapparates vor und bestimmte den Gang der Lichtabsorption im sichtbaren Spectrum zunächst qualitativ durch Beobachten der Lösungen in verschiedenen Concentrationen und in Schichten von verschiedener Dicke.

Es ergab sich, dass der von Herrn Dr. Friedländer frisch hergestellte salzsaure Diamidoindigo in seiner wässerigen Lösung ein deutliches, bei geringer Schichtendicke der blau gefärbten Lösung ziemlich schmales Absorptionsband in RothOrange besitzt, welches sich in größerer Concentration oder Schichtendicke rascher gegen Grün und Blau als gegen Roth verbreitet, wie dies Curve 2 in der Figur schematisch darstellt. Das 
Maximum der Lichtabsorption (Mitte des schmalen Streifens) lag nach meinen Versuchen bei der Wellenlänge $\lambda=623 \mu \mu^{1}$

Tetrazoindigo wurde durch Zusatz von Kaliumnitrit und etwas Salzsäure zur Lösung des salzsauren Diamidoindigo hergestellt; die blaue Farbe der letzteren schlägt hiebei in ein lebhaftes Rothviolett um. Im Spectrum treten dann zwei Absorptionsbänder auf, ein starkes $H$ a u p tband der Absorption, dessen Maximum bei $\lambda 565$ im Gelb liegt, während ein zweites

Indigodisulfosaures $\mathrm{Na}$

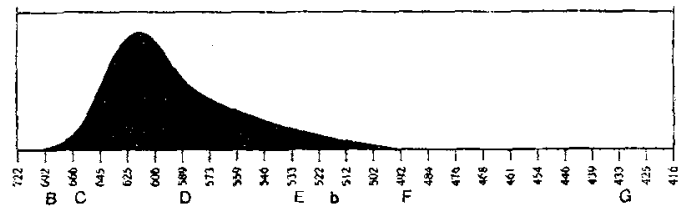

Diamidoindigo

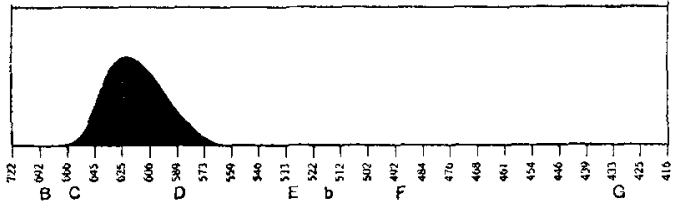

Tetrazoindigo

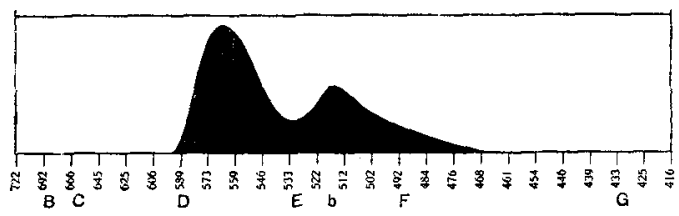

Absorptionsspectren, bezogen auf Fraunhofer'sche Linien und Wellenlängen.

schwächeres Nebenband der Absorption im Grün das Maximum bei $\lambda .517 \mu \mu$ besitzt. Diese getrennten Absorptionsbänder sind nur bei entsprechender Verdünnung sichtbar; bei großer Concentration verschwimmen beide Bänder zu einem intensiven, zusammenhängenden, breiten Absorptionsbande, während bei zu starker Verdünnung nur das Hauptabsorptions-

1 Krüss hat vor einer Reihe von Jahren gleichfalls ein Diamidoindigo spectroskopisch untersucht und fand die Lage des Absorptionsstreifens bei $588 \cdot 9$, jedoch ist das Krüss'sche Amidoindigo keineswegs identisch mit dem Friedländer'schen Amidoindigo, sondern letzterer ist ein anderer Körper. 
band allein übrig bleibt; in Curve 3 der Figur ist dies schematisch graphisch dargestellt.

Es ist von Interesse, diese optischen Eigenschaften von Diamidoindigo mit jenen des reinen Indigotins $z u$ vergleichen. Das Indigotin war zwar schon wiederholt Gegenstand spectralanalytischer Untersuchungen; namentlich C. H. Wolff ${ }^{1}$ untersuchte die Lösung des Indigotins in concentrierter Schwefelsäure; indes gestatten vergleichende Versuche verschiedener Substanzen mittels ein und desselben Apparates und desselben Beobachters größere Sicherheit der Vergleichung; deshalb unterzog ich die von Herrn Prof. Friedländer mir übergebene Lösung von indigodisulfosaurem Natrium, welche derselbe aus chemisch reinem Indigotin hergestelit hatte, gleichfalls einer Untersuchung. Bei genügend großer Verdünnung zeigt die Indigotinlösung ein ziemlich schmales Absorptionsband im Orange, dessen Mitte bei $\lambda 615$ liegt. Bei stärkerer Concentration rückt das Absorptionsband langsamer gegen Roth als gegen Blau vor, wie dies Curve 1 der Figur andeutet; bei noch größerer Concentration trübt die Indigoschwefelsäurelösung die stärker brechbaren Farben des Spectrums. Die Mitte des Maximums des in verdünnten Indigotinlösungen eben noch sichtbaren Absorptionsbandes befindet sich nach meinen wiederholten Versuchen bei $\lambda 615 .^{2}$

Um zur genaueren Kenntnis der optischen Eigenschaft des Indigotins und seiner Derivate zu gelangen, unterzog ich die oben erwähnten Lösungen einer quantitativen spectralanalytischen Untersuchung und bestimmte den Extinctionscoefficienten, sowie das Absorptionsverhältnis des Farbstoffes gemäß der Vierordt'schen Methode mittels Doppelspalt. ${ }^{3}$

1 Zeitschrift für analytische Chemie, Bd. 17, S. 310; Krüss, Colorimetrie und quantitative Spectralanalyse, 1890, S. 195.

1 H. W. Vogel, Prakt. Spertralanalyse irdischer Stoffe, 1889, S. 386 gibt an, dass der Absorptionsstreifen der Lösung von Indigo in concentrierter Schwefelsäure bei $\lambda 605$ liege, welche Zahl für das von mir untersuchte Indigotin sicherlich zu niedrig ist.

2 Traube, Physikalisch-chemische Methoden, 1893. Hiebei wird unter Concentration $c$ der Lösung die in $1 \mathrm{~cm}^{3}$ der Lösung enthaltene Anzahl Gramme der gelösten Substanz verstanden. 
a) Zunächst gieng ich von der Indigotinlösung aus, welche $\mathrm{mir}$ in einer Concentration von $0.0217 \mathrm{~g}$ Indigotin $=0.0375 \mathrm{~g}$ indigodisulfosaures Natrium $\mathrm{C}_{16} \mathrm{H}_{8} \mathrm{~N}_{2} \mathrm{O}_{2}\left(\mathrm{SO}_{3} \mathrm{Na}\right)_{2}$ in $1 \mathrm{~cm}^{3}$ vorlag; ich bestimmte die beim Lichtdurchgang durch die Farbstofflösung übrigbleibende Lichtstärke $\left(J^{\prime}\right)$ im Sinne Vierordt's, berechnete dementsprechend den Extinctionscoefficienten $e\left(e=-\log J^{\prime}\right)$ und leitete unter Berücksichtigung der Concentration der Lösung das sogenannte Absorptionsverhältnis $A\left(A=\frac{c}{e}\right)$ ab, da diese Größe eine für die gelöste Farbsubstanz charakteristische wichtige Constante ist. Die oben erwähnte Indigotinlösung brachte ich durch Verdünnung auf die Concentration von $0.00001085 \mathrm{~g}$ Indigotin, entsprechend $0.00001875 \mathrm{~g}$ indigodisulfosaurem Natrium in $1 \mathrm{~cm}^{3}$ Lösung, und untersuchte jene Region des Indigospectrums, in welcher das Maximum der Absorption liegt, d. i. nach meinen Befunden die Zone bei $\lambda 615$; diesen Beobachtungsbezirk variierte ich innerhalb der Grenzen der Zulässigkeit, bestimmte die dazu gehörigen Extinctionscoefficienten und fand bei 20 bis $23^{\circ} \mathrm{C}$.:

Lichtabsorption in indigodisulfosaurem Natrium $\left(0.00001875 \mathrm{~g}\left[\mathrm{C}_{16} \mathrm{H}_{8} \mathrm{~N}_{2} \mathrm{O}_{2}\left(\mathrm{SO}_{3} \mathrm{Na}\right)_{2}\right]\right.$ in $1 \mathrm{~cm}^{3}$ Lösung).

\begin{tabular}{|c|c|c|c|}
\hline $\begin{array}{c}\text { Spectralregion } \\
\mu \mu\end{array}$ & Lichtstärke & $\begin{array}{c}\text { Extinctions- } \\
\text { coefficient }\end{array}$ & $\begin{array}{c}\text { Absorptions- } \\
\text { verhältnis }\end{array}$ \\
\hline \hline $620-613$ & 0.21 & 0.67779 & 0.00002767 \\
$618-610$ & 0.19 & 0.72125 & 0.00002599 \\
$618-603$ & 0.20 & 0.69897 & 0.00002685 \\
\hline
\end{tabular}

Lichtabsorption in voriger Lösung von indigodisulfosaurem Natrium, berechnet auf Indigotin.

\begin{tabular}{|c|c|c|c|}
\hline $\begin{array}{c}\text { Spectralregion } \\
\mu \mu\end{array}$ & Lichtstärke & $\begin{array}{c}\text { Extinctions- } \\
\text { coefficient }\end{array}$ & $\begin{array}{c}\text { Absorptions- } \\
\text { verhältnis }\end{array}$ \\
\hline \hline $620-613$ & $0 \cdot 21$ & 0.67779 & 0.00001601 \\
$618-610$ & $0 \cdot 19$ & 0.72125 & 0.00001505 \\
$618-603$ & 0.20 & 0.69897 & 0.00001551 \\
\hline
\end{tabular}


Aus diesen Befunden ergibt sich, dass das Versuchsresultat selbstverständlich etwas von der Wahl der Spectralzone beeinflusst wird; ich halte für quantitative spectro-colorimetrische Bestimmungen den Spectralbezirk von $\lambda=618-610$ für den geeignetsten oder (falls der Beobachter nicht über die nöthige Übung verfügt, um in schmalen Spectralzonen gut messen zu können) den Bezirk von $\lambda=618-603$ für einen ebenfalls hiefür geeigneten Spectralbezirk.

Diese meine Befunde über das Absorptionsverhältnis des Indigotins stimmen befriedigend mit dem von Wolff zum Zwecke der spectralanalytischen Wertbestimmung des Indigos für reines Indigotin ermittelten Absorptionsverhältnis, wenn man berücksichtigt, dass letzterer seine Bestimmung nicht in ganz demselben Spectralbezirk, sondern für die Spectralbezirke $C 65 D-C 90 D$, d. i. $\lambda=613-596$ machte $^{1}$ und dabei $A=0.0000142$ fand.

Auf Grund meiner eigenen Untersuchungen setze ich das charakteristische Absorptionsverhältnis für reines Indigotin in Form von indigodisulfosaurem Natrium für die Region $\lambda=618-610 A=0.00002599$, oder berechnet auf Indigotin $A=0.00001505$.

b) Der Diamidoindigo Friedländer's besitzt in der Region seiner größten Lichtabsorption, d. i. bei $\lambda=626-618$ bei einer Concentration von $(0.0000217 \mathrm{~g}$ Acetamidoindigo $\Rightarrow)$ $0 \cdot 0000167 \mathrm{~g}$ Amidoindigo in $1 \mathrm{~cm}^{3}$ Lösung folgende Zahlen:

Lichtabsorption von Amidoindigo.

\begin{tabular}{|c|c|c|c|c|}
\hline & $\begin{array}{c}\text { Spectral- } \\
\text { region }\end{array}$ & $\begin{array}{c}\text { Licht- } \\
\text { stärke }\end{array}$ & $\begin{array}{c}\text { Extinctions- } \\
\text { coefficient }\end{array}$ & $\begin{array}{c}\text { Absorptions- } \\
\text { verhältnis }\end{array}$ \\
\hline $\begin{array}{c}\text { Acetamidoindigo ... } \\
\text { Berechnet auf Amido- } \\
\text { indigo ......... }\end{array}$ & $\lambda 626-618$ & 0.315 & 0.50169 & 0.00004325 \\
\hline
\end{tabular}

1 Die veraltete Vierordt'sche Bezeichnung der Spectralbezirke durch Eintheilen der Zwischenräume zwischen den Fraunhofer'schen Hauptlinien kann mittels der von Krüss (a. a. O. S. 289) angegebenen Tabellen auf Wellenlängen umgerechnet werden. Weitaus sicherer ist die directe Aichung der Scala des Spectralapparates auf Wellenlängen, wie dies auch bei meinen Untersuchungen geschah. 
c) Tetrazoindigo. Führt man eine Diamidoindigolösung nach Prof. Friedländer's Vorgang in Tetrazoindigolösung über, so gewinnt das Hauptband (siehe oben) im Absorptionsspectrum der rothvioletten Lösung eine merklich stärkere Absorption (größere Extinctionscoefficienten) als die Diamidoindigolösung größerer Concentration, aus welcher die Tetrazoverbindung hervorgieng, 1 besaß. Dagegen weist das zweite auftretende Absorptionsband einen wesentlich geringeren Extinctionscoefficienten auf; dies ist aus den in nachfolgender Tabelle angegebenen Messungen ersichtlich.

Lichtabsorption der salzsauren Tetrazoindigolösung.

\begin{tabular}{|c|c|c|c|c|}
\hline & $\begin{array}{c}\text { Spectral- } \\
\text { bezirk } \\
\mu \mu\end{array}$ & $\begin{array}{c}\text { Licht- } \\
\text { stärke }\end{array}$ & $\begin{array}{c}\text { Extinctions- } \\
\text { coefficient }\end{array}$ & $\begin{array}{c}\text { Absorptions- } \\
\text { verhältnis }\end{array}$ \\
\hline \hline Hauptband $\ldots \ldots \ldots$ & $567-561$ & 0.222 & 0.65758 & 0.00003376 \\
Nebenband ...... & $518-515$ & $0.19^{3}$ & 0.72125 & 0.00007695 \\
\hline
\end{tabular}

Aus diesen Befunden geht hervor, dass das Maximum des Absorptionsbandes im Indigoschwefelsäurespectrum bei der Wellenlänge $\lambda=615$ liegt, durch Einführung der A midogruppe gegen Roth etwas verschoben wird (um $8 \mu \mu$ ), so dass es auf $\lambda=623 \mathrm{zu}$ liegen kommt. Durch Überführung des Diamidoindigo in das entsprechende salzsaure Tetrazoin digo wird das einfache Absorptionsband im Orange in ein gelbes und grünes Absorptionsband (bei $\lambda=565$ und 517) gespalten.

An der Stelle der betreffenden Absorptionsbänder ergeben sich die oben angegebenen charakteristischen Absorptionsverhältnisse, welche zur quantitativen spectro-colorimetrischen Bestimmung dieser Farbstoffe geeignet sind.

1 Dabei ist hypothetisch angenommen, dass die Friedländer'sche Umsetzung von Amidoindigo in Tetrazoindigo quantitativ sich volizieht.

2 Concentration $0.0000222 \mathrm{~g}$ salzsaures Tetrazoindigo, entsprechend $0.0000167 \mathrm{~g}$ Diamidoindigo $=0.0000217 \mathrm{~g}$ Acetamidoindigo in $1 \mathrm{~cm}^{3}$ Lösung.

3 Concentration entsprechend $0.0000555 \mathrm{~g}$ salzsaures Tetrazoindigo in $1 \mathrm{~cm}^{3}$ Lösung.

4 Berechnet auf salzsaures Tetrazoindigo, von welchem $0.0000222 \mathrm{~g}$ $=0.0000217 \mathrm{~g}$ Acetamidoindigo sind. 